\title{
Journal of Clinical Case Studies
} Inhibitors on Endometriosis-Related Pain and Endometrial Proliferation-A Short Study. J Clin Case

\author{
H Maia Jr ${ }^{1 *}$, C Haddad ${ }^{1}$ and W Saback Junior ${ }^{2}$
}

'Instituto da Mulher, Itaigara Memorial Day Hospital, Salvador, Bahia, Brazil

${ }^{2}$ Department of Life Sciences, State University of Bahia (UNEB), Salvador, Bahia, Brazil Stu 3(1): doi http://dx.doi.org/10.16966/2471-4925.161

Copyright: ( 2017 Maia Jr H, et al. This is an openaccess article distributed under the terms of the Creative Commons Attribution License, which permits unrestricted use, distribution, and reproduction in any

*Corresponding author: H Maia Jr, Instituto da Mulher, Itaigara Memorial Day Hospital, Salvador, Bahia, Brazil, E-mail: hmaiaf@terra.com.br medium, provided the original author and source are credited.

\begin{abstract}
Objective: The present study was designed to investigate the effects of valproic acid or resveratrol, together with vitamin D3 (VITD3) in Pentravan ${ }^{\circledR}$ (PTV) administered vaginally, on deep endometriosis-associated pain in patients undergoing treatment with gestrinone.

Material and methods: This was an open study involving 30 patients with deep endometriosis and pelvic pain unresponsive to previous progestin-based treatment. Pain was assessed at the initial visit and after the first and third months of treatment using a visual analog scale that graded pain from 0 to 10 . Hysteroscopy with endometrial biopsy $(n=21)$ was performed at baseline and after the first treatment month to determine aromatase expression by immunohistochemistry. Patients were divided into three groups. Patients in Group I $(n=16)$ were treated for 6 months with vaginal gestrinone (GTN) $(5 \mathrm{mg} / \mathrm{ml})$ twice weekly together with pycnogenol $(100 \mathrm{mg})$ and silymarin $(400 \mathrm{mg})$ administered orally once a day. In Group II $(\mathrm{n}=8)$, patients received the same treatment together with vaginal resveratrol $(100 \mathrm{mg} / \mathrm{ml})$ and vitamin D3 $(5000 \mathrm{U} / \mathrm{ml})$ administered daily. Patients in Group III $(n=9)$ were treated with gestrinone together vaginal valproic acid (VPA) (250 mg) administered daily. All vaginal medications were prepared in Pentravan ${ }^{\circledR}$.

Results: There was a significant decrease in the mean pain score of patients in Group I from 9 at baseline to 3 after the first month of treatment. In Groups II and III, on the other hand, the pain score decreased from a mean of 9 to a mean of 1, a significantly greater decrease than that achieved in Group I $(p=0.01)$. Although the reduction in pain was significant in all the groups, after the first month of treatment it was much greater in the groups treated simultaneously with histone deacetylase (HDAC) inhibitors. By the third month of treatment, all patients in all three groups were amenorrheic and pain-free, with no difference between the groups. After the first treatment month, aromatase expression remained positive in the endometrium of $8 / 10$ women $(80 \%)$ in Group I compared to only $1 / 8(12 \%)$ in Group II $(p=0.006)$ and $2 / 3(66 \%)$ in Group III (p=0.04 compared to Group II but not significantly different from Group I).

Conclusion: The use of HDAC inhibitors in Pentravan ${ }^{\circledR}$ potentiated the pain-relieving effects of gestrinone in patients with deep endometriosis in the first month of treatment; however, this difference disappeared in the subsequent months. This suggests that if much faster pain relief were desired in cases of deep endometriosis, then combining vaginal gestrinone with an HDAC inhibitor would be preferable to using gestrinone alone. The combined use of gestrinone with epigenetic drugs is a novel and highly effective treatment for deep endometriosis-related pain.
\end{abstract}

\section{Introduction}

Accumulating evidence suggests that endometriosis is an epigenetic disease of the endometrium in which a great number of genes are either abnormally activated or silenced through either DNA methylation or histone acetylation [1]. Aberrant histone modifications are present in endometriosis, since levels of histone acetylation were significantly higher in the endometriotic stromal cells compared to the normal endometrium [2]. The process of histone acetylation is regulated by the balance between the activity of the histone acetyltransferases and HDACs. The interplay between the activities of these two enzymes is thought to play a crucial role in the regulation of gene transcription by remodeling chromatin structure [3]. The addition of acetyl groups to histones loosens the DNA packing and permits gene transcription to occur, while the reverse leads to gene silencing. Since the levels of histone acetylation are lower in endometriosis, the use of HDACs inhibitors to suppress histone deacetylation and activate silenced genesmay constitutes a novel therapeutic approach for the treatment of endometriosis.

Resveratrol (RVT), an HDAC inhibitor had been previously shown to potentiate the pain-relieving effects of continuous oral contraceptives on endometriosis-associated pain and this was associated with a greater inhibition of aromatase expression in the eutopic endometrium [4,5].
In these initial studies, resveratrol was used orally. Aberrant aromatase expression occurs concomitantly in both the eutopic endometrium and in the endometriotic lesions, and can be considered an instigator of the epigenetic changes occurring in this disease, since it is a consequence of the hypo-methylation of the promoter gene for this enzyme. The persistence of aromatase expression may therefore be used as a marker of progesterone resistance [5]. In endometriosis, it is also common for various degrees of progesterone resistance to occur, caused by the partial silencing of the progesterone receptor-B gene, thus hampering medical treatment with continuous progestins or oral contraceptives in an extended regimen [6]. This results in the recurrence of pain and menstrual-like bleeding, leading to treatment failure and leaving repeat surgeries as one of the alternatives for these patients.

More recently, the use of a 19 nor-testosterone (19NT) derivative, gestrinone (GTN), administered by the vaginal route in Pentravan in association with oral RVT, proved highly effective for the treatment of endometriosis-related pain in patients who failed to respond to progestin therapy [7]. In these patients, the combined use of vaginal gestrinone with oral resveratrol and Pinus pinaster extract rendered them pain-free by the end of the third month of treatment. Although total pain scores had already decreased significantly after one month of gestrinone treatment, 
most patients still report pain at the completion of the first month of therapy, although the intensity of the pain is significantly less. Since these patients are chronic pain sufferers, if a treatment could be designed that would render them pain-free within a shorter period of time, this would certainly improve compliance with treatment. One possibility would be to combine gestrinone treatment with vaginal resveratrol and vitamin D3 in Pentravan to reverse the epigenetic changes that favor the development of endometriosis, while concomitantly blocking aromatase NF-Kappa B activation. Theoretically, this would result in a much faster reduction in pain. The combination of resveratrol and vitamin D3might exert a much stronger inhibitory effect on inflammation in the endometriosis lesions, since resveratrol increases the number of vitamin D3 receptors, thus enhancing their anti-inflammatory action [8].

Another HDAC inhibitor currently used for the treatment of epilepsy and bipolar disorders is valproicacid (VPA). This medication has been previously used to treat pain in patients with uterine fibroids and endometriosis in conjunction with the levonorgestrel-releasing intrauterine system (Mirena") [9]. However, neither VPA nor RVT with vitamin D3 (VTD3) has been used before in Pentravan for vaginal use. Theoretically, the use of gestrinone associated with HDAC inhibitors would lead to a much faster reduction in pain scores. The vaginal administration of either valproic acid or resveratrol with vitamin D3 together with gestrinone should increase the efficacy of the latter in reducing the painful symptoms associated with endometriosis, since this form of treatment would result in a faster reversal of the epigenetic changes that favor the development of endometriosis.

The present study was conducted to evaluate the effects of valproic acid or resveratrol with VIT D3, administered through the vaginal mucosa in Pentravan, on pain scores of patients with endometriosis undergoing treatment with GTN. The vaginal administration of with HDAC inhibitors was expected to be more effective by concentrating these drugs in the pelvis because of the phenomenon of the first uterine passage [10].

\section{Patient and Methods}

This was an open study involving 30 patients of reproductive age (range 20-42 years) with deep endometriosis. The inclusion criteria were the presence of severe intractable pelvic pain. Previous treatment with dienogest or oral contraceptives was not exclusion criteria if the treatment had been discontinued three months prior to the commencement of the study. All patients were of reproductive age (20-45 year old), nulliparous and were not desiring to get pregnant in a near future since the primary end point of the study was pain resolution and not fertility. Previous surgical treatment was not an exclusion criteria if pain had recurred at least one year following surgery. Endometriosis patients with associated uterine pathologies diagnosed by transvaginal ultrasonography such as were uterine fibroids $(n=13)$ or and adenomyosis $(n=13)$ were also included in the study.

The patients were initially interviewed by the same investigator (HM) and pain was assessed using a visual analogic scale (VAS) in which zero corresponded to the total absence of pain and ten to the worst pain imaginable [11]. A total pain score was calculated, which included dysmenorrhea, pelvic pain and dyschesia. When the patients gave different VAS scores for these different forms of pain, the one with the highest score was considered for the purpose of evaluation. Pain was assessed at the initial visit and again after the first and third months of treatment. Transvaginal ultrasonography was performed at baseline and again when the patients completed one month of treatment. Hysteroscopy with endometrial biopsy $(n=21)$ was performed prior to and after one month of treatment to determine aromatase expression by immunohistochemistry. Patients whose pretreatment endometrial biopsy tested positive for aromatase expression were submitted to a second hysteroscopy procedure irrespective of the treatment they were given.
The patients were divided into three groups. Although pain scores were similar at baseline, allocation of the patients to the individual groups was not randomized. The study was open in that both the patients and their attending physician were aware of the treatment. In Group I $(n=16)$, patients were treated for six months with vaginal gestrinone $(5 \mathrm{mg} / \mathrm{ml})$ in Pentravan twice weekly, together with pycnogenol $(100 \mathrm{mg})$ and silimarin $(400 \mathrm{mg})$ taken daily by the oral route. Six of the patients in this group (37\%) had uterine fibroids as well as endometriosis, and seven had concomitant adenomyosis (43\%). In group II $(n=8)$, the patients received the same treatment with gestrinone as those in Group I, together with the daily vaginal administration of resveratrol $(100 \mathrm{mg} / \mathrm{ml})$ and vitamin D3 (5000 U/ml) in Pentravan'. As with Group I, Pinus pinaster (100 mg/day) and silimarin were used orally. Four patients in this group had uterine fibroids (50\%) and three had adenomyosis (37\%). In Group III ( $n=6)$, the patients received the same gestrinone treatment as those in the previous groups, but valproic acid $(250 \mathrm{mg})$ in Pentravan was also used daily by the vaginal route. Three patients in this group also had uterine fibroids (50\%) and three had adenomyosis (50\%) in addition to endometriosis.

Statistical analysis was performed using Student's t-test for paired samples to evaluate pain scores and the chi-square test to evaluate differences in percentages, with $\mathrm{p}$-values $<0.05$ being considered statistically significant. However tests for normality of distribution were not applied since the number of enrolled patients was small in all groups.

The study was conducted at the Instituto da Mulher, Itaigara Memorial Day Hospital and was approved by the institute's internal review board. All patients enrolled gave informed consent after being explained of the purpose of the study was to alleviate pain in this patient population which had failed to respond to other hormonal therapies.

\section{Results}

\section{Effect on pain scores}

In Group I, the combined treatment of vaginal gestrinone in Pentravan associated with oral silimarin and Pinus pinaster extract resulted in a significant decrease in the mean pain score from a mean of 9 at baseline to 3 after the first month of treatment. In Groups II and III, on the other hand, the use of vaginal VIT D3 with resveratrol or VLA led to a greater decrease in the mean pain score after 30 days of treatment compared to Group I. In both Groups II and III, the decrease in overall pain was greater than in the group using gestrinone alone, reaching a mean score of 1 , which was significantly lower than the mean pain score of patients in Group I $(\mathrm{p}=0.01)$. Although the pain reduction was significant in all groups, it was much greater in the groups treated simultaneously with HDAC inhibitors after the first month of treatment. On the other hand, by the third month of gestrinone treatment, the patients in all three groups were amenorrheic and pain-free and they remained so throughout treatment (Figure 1). The overall reduction in pain included not only dysmenorrhea but also the other forms of pain such as dyspareunia and dyschesia. At 6 months, all the patients in all the groups still remained pain-free.

\section{Effect of Vitamin D3 on aromatase expression}

In patients not using vaginal GNT alone (Group I) in whom hysteroscopy was carried out between 20 and 30 days after the initiation of treatment aromatase expression was still detected in $80 \%$ of the patients $(8 / 10)$. In patients using vaginal resveratrol with vitamin D3 (Group II) in Pentravan, on the other hand, only $12 \%$ showed positive aromatase expression in the endometrium, a percentage that was significantly lower than that of Group I ( $80 \%)(p=0.006)$. In Group III, aromatase expression was detected in the first month in the endometrium of $2 / 3$ patients $(66 \%)$. There was a statistically significant difference between this percentage and that of Group II $(p=0.04)$ but there was no statistically significant difference between the percentages in Group III and Group I (Figure 2). 

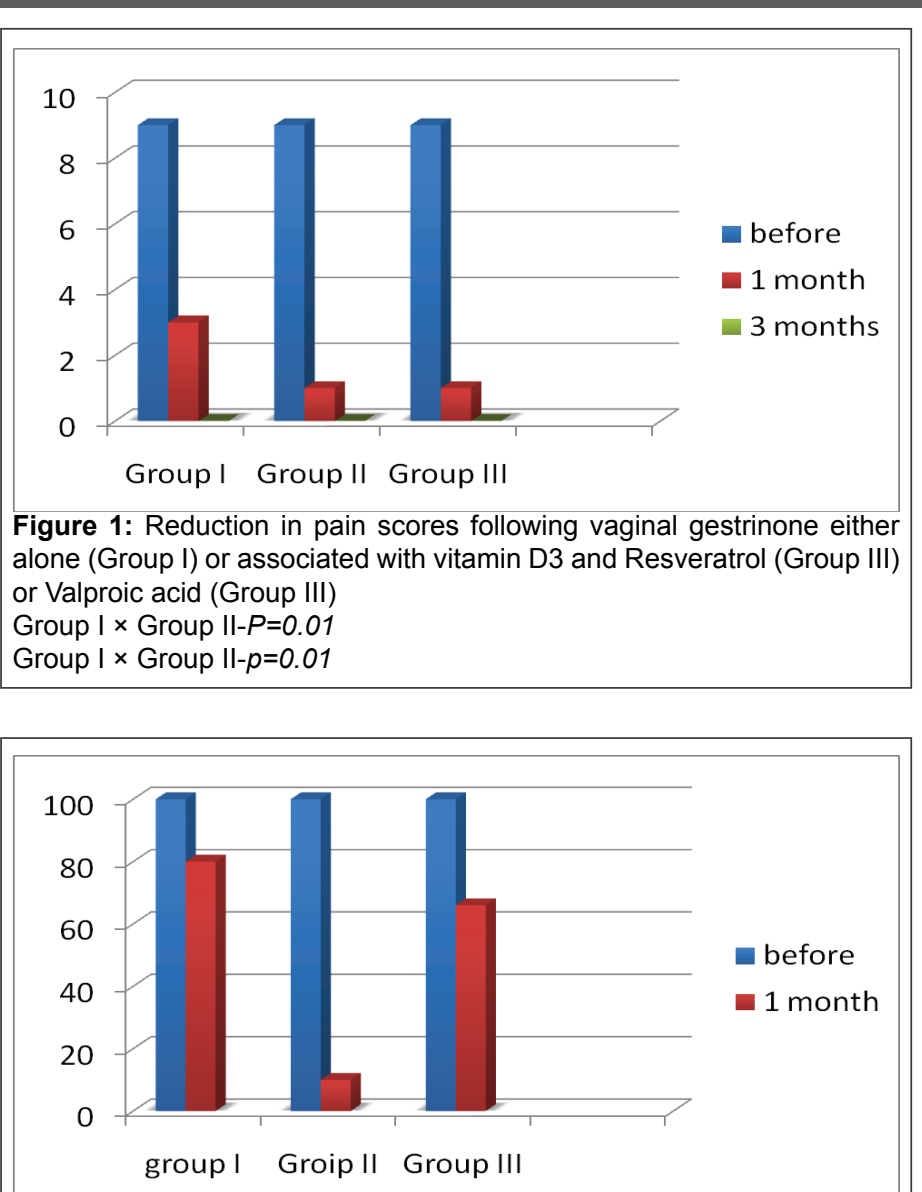

Figure 2: Effect of vaginal vitamin D3 + Resveratrol or Valproic acid in pentravan on aromatase expression in endometriosis patients using vaginal gestrinone

Group I $\times$ Group II- $p=0.006$

Group II $\times$ Group III $p=0.04$

Group I × Group III NS

\section{Discussion}

The present results show that the use of vaginal HDAC inhibitors potentiated the pain-relieving effects of gestrinone for the treatment of endometriosis-related pain in the first month of treatment. There was a significantly greater reduction in mean pain scores in Groups II and III compared to Group I; however, this difference dissipated in the subsequent months. This suggests that if a much faster reduction in pain were desirable in patients with deep endometriosis, then a combination of gestrinone with an HDAC inhibitor would be the treatment of choice. These initial results suggest that HDAC inhibitors may exert a potentiating effect on pain circuits there, which is in agreement with the findings of previous studies on the role of epigenetic changes associated with pain resulting from an inflammatory process [12]. Histone deacetylase (HDAC) inhibitors such as VPA acid or RVT act by preventing the removal of acetyl groups from histones, which have successfully improved the symptoms of pain in a number of animal models used for studying inflammatory diseases such as arthritis, colitis and hepatitis [13-15]. Endometriosis is also a typical inflammatory pathology, which would explain the prompt response achieved in terms of pain relief when HDAC inhibitors are used concomitantly with gestrinone.

The present results are also in agreement with previous findings showing an increase in HDAC activity in patients suffering with endometriosis and endometriosis-associated pain [1-3]. There is no doubt that there is a potential for the study of novel drug targets for the treatment of endometriosis-related pain. It is already clear from the few experiments that have already been conducted that HDAC inhibitors could be an interesting group of drugs to be tested in chronic pain sufferers. Currently, the selectivity of compounds such as VPA is limited and, consequently, if given systemically, side effects such as fatigue, nausea and diarrhea may occur. The use of the vaginal route, as reported here, as a means of administering VPA in Pentravan', may eliminate some of these unpleasant side effects while also potentiating the pain-relieving effects of gestrinone in patients with deep endometriosis. To the best of our knowledge this is the first report, albeit preliminary showing the effectiveness of VPA when used vaginally in Pentravan " to potentiate the analgesic effects of GTN in deep endometriosis patients.

Because of their relevance in the fight against cancer, the development of more selective and hence more tolerable HDAC inhibitors is a high priority, not only for research but also for the pharmaceutical industry [3]. With respect to pain, drugs that specifically block HDAC activity such as RVT or VPA would be of particular interest. There is some evidence suggesting that HDAC inhibitors may be involved in pain processing, since they are able to decrease inflammatory cytokines, thus relieving pain by decreasing inflammation [15].

The use of VIT D3 with RVT also potentiated the inhibitory effect of GTN on aromatase expression in the endometrium of patients with endometriosis, thus confirming previous studies on the inhibitory effects of these substances on the expression of this enzyme [16]. VIT D3 may exert a pain-relieving effect on dysmenorrhea and this effect may be potentiated by the concomitant use of RVT resveratrol, which stimulates the synthesis of vitamin D3 receptors (VDR) [8].

In conclusion, the association of resveratrol with VITD3 or VPA, together with gestrinone, administered vaginally in Pentravan', decreased endometriosis-associated pain in the first three months of treatment more effectively than the use of gestrinone alone; however, these differences dissipate after the third month of treatment. However due to the small sample of the study these results have to be considered preliminary and further larger trials are required to corroborate these findings. Despite these limitations our findings suggest that it is possible to use the vaginal route to administer gestrinone associated with other drugs in Pentravan" in order to treat endometriosis pain thus postponing the need for more invasive surgical procedures. One pitfall of this study, however, is the lack of data on the Pharmacodynamics of the used drugs so it is not possible to infer whether the best dosage or time of administration was used. The prompt response in terms of pain resolution in our group of patients with deep endometriosis supports previous studies on a biological plausibility for a role of VIT D3 and its receptor in suppressing inflammation in both eutopic and ectopic endometrium. The expression of the VDR and enzymes involved in VIT D3 metabolism also show dysregulation in endometriosis lesions when in animal models [16]. In mice, on the other hand, Elocalcitol a VDR agonist was shown to reduce the development and recurrence of endometriosis lesions [17]. Similarly in rats the administration of VPA together with progesterone significantly reduced endometriosis lesions and decreased its associated hyperalgesia [18]. These animal studies are in concordance with our findings showing that both HDAC inhibitors and VIT D3 with RVT may be promising agents to decrease hyperalgesia associated with deep endometriosis when used vaginally in Pentravan" in patients undergoing treatment with GTN.

\section{References}

1. Nasu K, Kawano Y, Tsukamoto Y, Takano M, Takai N, et al. (2011) Aberrant DNA methylation status of endometriosis: epigenetics as the pathogenesis, biomarker and therapeutic target. J Obstet Gynaecol Res 37: 683-695. 
2. Kawano Y, Nasu K, Li H, Tsuno A, Abe W, et al. (2011) Application of the histone deacetylase inhibitors for the treatment of endometriosis: histone modifications as pathogenesis and novel therapeutic target. Hum Reprod 26: 2486-2498.

3. Marks PA, Rifkind RA, Richon VM, Breslow R (2001) Inhibitors of histone deacetylase are potentially effective anticancer agents. Clin Cancer Res 7: 759-760.

4. Maia H Jr, Haddad C, Pinheiro N, Casoy J (2012) Advantages of the association of resveratrol with oral contraceptives for management of endometriosis-related pain. Int J Womens Health 4: 543-549.

5. Maia H Jr, Haddad C, Coelho G, Casoy J (2012) Role of inflammation and aromatase expression in the eutopic endometrium and its relationship with the development of endometriosis. Womens Health (Lond) 8: 647-658.

6. Patel BG, Rudnicki M, Yu J, Shu Y, Taylor RN (2017) Progesterone resistance in endometriosis: origins, consequences and interventions. Acta Obstet Gynecol Scand 96: 623-632.

7. Maia H Jr, Haddad C, Moura Hirsch MCD, Saback W, Casoy J (2014) Treatment of Refractory Endometriosis-Related Pain with Vaginal Gestrinone in Pentravan Associated with Pinus Pinaster Extract and Resveratrol: A Preliminary Study. Gynecol Obstet (Sunnyvale) 4: 246.

8. Dampf Stone A, Batie SF, Sabir MS, Jacobs ET, Lee JH, et al. (2015) Resveratrol potentiates vitamin $\mathrm{D}$ and nuclear receptor signaling. $\mathrm{J}$ Cell Biochem 116: 1130-1143.

9. Xishi Liu, Lei Yuan, Guo SW (2010) Valproic acid as a therapy for adenomyosis: a comparative case series. Reprod Sci 17: 904-912.

10. De Ziegler D, Bulletti C, De Monstier B, Jääskeläinen AS (1997) The first uterine pass effect. Ann N Y Acad Sci 828: 291-299.
11. Kelly AM (1998) Does the clinically significant difference in visual analog scale pain scores vary with gender, age, or cause of pain? Acad Emerg Med 5: 1086-1090.

12. Selvi BR, Mohankrishna DV, Ostwal YB, Kundu TK (2010) Small molecule modulators of histone acetylation and methylation: a disease perspective. Biochim Biophys Acta 1799: 810-828.

13. Chung YL, Lee MY, Wang AJ, Yao LF (2003) A therapeutic strategy uses histone deacetylase inhibitors to modulate the expression of genes involved in the pathogenesis of rheumatoid arthritis. Mol Ther 8:707-717.

14. Glauben R, Batra A, Fedke I, Zeitz M, Lehr HA, et al. (2006) Histone hyperacetylation is associated with amelioration of experimental colitis in mice. J Immunol 176: 5015-5022.

15. Leoni F, Zaliani A, Bertolini G, Porro G, Pagani P, et al. (2002) The antitumor histone deacetylase inhibitor suberoylanilide hydroxamic acid exhibits antiinflammatory properties via suppression of cytokines. Proc Natl Acad Sci U S A 99: 2995-3000.

16. Sayegh L, Fuleihan Gel-H, Nassar AH (2014) Vitamin D in endometriosis: a causative or confounding factor? Metabolism 63: $32-41$.

17. Mariani M, Viganò P, Gentilini D, Camisa B, Caporizzo E, et al. (2012) The selective vitamin D receptor agonist, elocalcitol, reduces endometriosis development in a mouse model by inhibiting peritoneal inflammation. Hum Reprod 27: 2010-2019.

18. Liu M, Liu X, Zhang Y, Guo SW (2012) Valproic acid and progestin inhibit lesion growth and reduce hyperalgesia in experimentally induced endometriosis in rats. Reprod Sci 19: 360. 